\title{
Cubic B-spline Collocation Method for One-Dimensional Heat Equation
}

\author{
Mohamed Hassan Khabir ${ }^{1}$, Rahma Abdullah Farah ${ }^{2}$ \\ ${ }^{1}$ Department of Mathematics, Faculty of Science, Sudan University of Science \& Technology, Khartoum, Sudan \\ ${ }^{2}$ Department of Mathematics, Faculty of Science \& Technology, Omdurman Islamic University, Khartoum, Sudan
}

Email address:

khabir11@gmail.com (M. H. Khabir),rahma999@windowslive.com (R. A. Farah)

\section{To cite this article:}

Mohamed Hassan Khabir, Rahma Abdullah Farah. Cubic B-Spline Collocation Method for One-Dimensional Heat Equation. Pure and Applied Mathematics Journal. Vol. 6, No. 1, 2017, pp. 51-58. doi: 10.11648/j.pamj.20170601.17

Received: November 26, 2016; Accepted: January 16, 2017; Published: March 4, 2017

\begin{abstract}
In this paper we discuss cubic B-spline collocation method. We have given the derivation of the B-spline method in general. We have applied the method for solving one-dimensional heat equation and the numerical result have been compared with the exact solution.
\end{abstract}

Keywords: Cubic B-spline, Collocation Method, Heat Equation, Linear Partial Differential Equation

\section{Introduction}

Consider the one dimensional initial-boundary value problem

$$
P_{r}=\left\{\begin{array}{l}
\frac{\partial u}{\partial t}=c \frac{\partial^{2} u}{\partial x^{2}}, c=1 \\
u(0, t)=0, u(1, t)=0(B . C S) \\
u(x, 0)=g(x)(I . C)
\end{array}\right.
$$

This problem is one of the well-known second order parabolic linear partial differential equation [1, 3, 4]. The heat equation is a very important equation in physics and engineering. It shows that heat equation describes the distribution of heat (or variation in temperature) in a given region over time. The heat equation is of fundamental importance in diverse scientific fields. In mathematics, it is prototypical parabolic partial differential equation. In probability theory, the heat equation is connected with the study of Brownian motion via the Fokker - Planck equation [5]. Numerical solutions of those equations are very useful to study physical phenomena. One of the linear evolution equation which we deal with the numerical solution is the heat equation [2]. In financial mathematics it is used to solve the Black - Scholes partial differential equation. The diffusion equation, a more general version of the heat equation, arises in connection with the study of chemical diffusion and other related processes [5]. In history, the heat equation proposed by Fourier in 1822 has been applied to investigating a temperature distribution in materials [6]. The heat equation is used in probability and describes random walks. It is also applied in financial mathematics for this reason. It is also important in Riemannian geometry and thus topology: it was adapted by Richard S. Hamilton when he defined the Ricci flow that was later used by Grigori Perelman to solve the topological Poincaré conjecture. The heat equation arises in the modeling of a number of phenomena and is often used in financial mathematics in the modeling of options. The famous Black - Scholes option pricing model's differential equation can be transformed into the heat equation allowing relatively easy solutions from a familiar body of mathematics. Many of the extensions to the simple option models do not have closed form solutions and thus must be solved numerically to obtain a modeled option price. The equation describing pressure diffusion in a porous medium is identical in form with the heat equation. Diffusion problems dealing with Dirichlet, Neumann and Robin boundary conditions have closed form analytic solutions (Thambynayagam 2011). The heat equation is also widely used in image analysis (Perona \& Malik 1990) and in machine-learning as the driving theory behind scale-space or graph Laplacian methods. The heat equation can be efficiently solved numerically using the implicit Crank Nicolson method of (Crank \& Nicolson 1947). This method can be extended to many of the models with no closed form solution, see for instance (Wilmott, Howison \& Dewynne 
1995). An abstract form of heat equation on manifolds provides a major approach to the Atiyah - Singer index theorem, and has led to much further work on heat equations in Riemannian geometry [5].

In this study the cubic B-spline collocation method is used $[7,9,10]$ for solving the heat equation (1) and the solutions are compared with the exact solution. In the section two, we have given the derivation for the B-spline method and uniform convergence for the method has been discussed. Finally, we have solved the problem (1) using the method, the numerical results and graphs have also been shown.

\section{B-spline Collocation Method}

We define the cubic B-spline for $i=0,1,2, \ldots, N$.

$$
B_{i}(x)= \begin{cases}\left(\frac{x-x_{i-2}}{\widetilde{h}}\right)^{3}, & \text { if } x \in\left[x_{i-2}, x_{i-1}\right], \\ 1+3\left(\frac{x-x_{i-1}}{\widetilde{h}}\right)+3\left(\frac{x-x_{i-1}}{\widetilde{h}}\right)^{2}+\left(\frac{x-x_{i-1}}{\widetilde{h}}\right)^{3}, & \text { if } x \in\left[x_{i-1}, x_{i}\right], \\ 1+3\left(\frac{x_{i+1}-x}{\widetilde{h}}\right)+3\left(\frac{x_{i+1}-x}{\widetilde{h}}\right)^{2}+\left(\frac{x_{i+1}-x}{\widetilde{h}}\right)^{3}, & \text { if } x \in\left[x_{i}, x_{i+1}\right], \\ \left(\frac{x_{i+2}-x}{\widetilde{n}}\right)^{3}, & \text { if } x \in\left[x_{i+1}, x_{i+2}\right], \\ 0, & \text { otherwise, }\end{cases}
$$

where $\tilde{h}=x_{i+1}-x_{i}, i=-1,0, \ldots, N+1$.

We introduce four additional knots as $x_{-2}<x_{-1}<x_{0}$ and $x_{N+2}>x_{N+1}>x_{N}$.

From the above Eq. (2) we can simply check that each of the functions $B_{i}(x)$ is twice continuously differentiable on the entire real line, also

$$
B_{i}\left(x_{j}\right)= \begin{cases}4, & \text { if } i=j \\ 1, & \text { if } i-j= \pm 1 \\ 0, & \text { if } i-j= \pm 2\end{cases}
$$

and that $B_{i}(x)=0$ for $x \geq x_{i+2}$ and $x \leq x_{i-2}$.

Similarly we can show that

$$
B_{i}^{\prime}\left(x_{\mathrm{j}}\right)= \begin{cases}0, & \text { if } i=j \\ \pm \frac{3}{\widetilde{h}}, & \text { if } i-j= \pm 1 \\ 0, & \text { if } i-j= \pm 2\end{cases}
$$

and

$$
B_{i}^{\prime \prime}\left(x_{j}\right)= \begin{cases}-\frac{12}{\widetilde{h}^{2}}, & \text { if } i=j \\ \frac{6}{\widetilde{h}^{2}}, & \text { if } i-j= \pm 1 \\ 0, & \text { if } i-j= \pm 2\end{cases}
$$

Each $B_{i}(x)$ is also a piece-wise cubic with knots at $\pi$, and $B_{i}(x) \in X$.

The values of $B_{i}(x), B_{i}^{\prime}(x)$ and $B_{i}^{\prime \prime}(x)$ at the nodal points $x_{i}{ }^{\prime} s$ are shown in Table 1 .

Table 1. B-Spline basis values.

\begin{tabular}{llllll}
\hline $\boldsymbol{x}$ & $\boldsymbol{x}_{\boldsymbol{i}-2}$ & $\boldsymbol{x}_{\boldsymbol{i}-1}$ & $\boldsymbol{x}_{\boldsymbol{i}}$ & $\boldsymbol{x}_{\boldsymbol{i}+\mathbf{1}}$ & $\boldsymbol{x}_{\boldsymbol{i}+\mathbf{2}}$ \\
\hline$B_{i}(x)$ & 0 & 1 & 4 & 1 & \\
$\tilde{h} B_{i}^{\prime}(x)$ & 0 & -3 & 0 & 3 & 0 \\
$\tilde{h}^{2} B_{i}^{\prime \prime}(x)$ & 0 & 6 & -12 & 6 & 0 \\
\hline
\end{tabular}

Nodal values

Let $\Omega=\left\{B_{-1}, B_{0}, B_{1}, \ldots, B_{N+1}\right\}$ and let $\phi_{3}(\pi)=$ span $\Omega$. The functions $\Omega$ are linearly independent on $[0,1]$, thus $\phi_{3}(\pi)$ is $(N+3)$-dimensional. Even one can show that $\phi_{3}(\pi) \subseteq_{s p} X$. Let $L$ be a linear operator with domain $X$ and with range in $X$.

Now we define

$$
\begin{gathered}
S(x)=\sum_{i=-1}^{N+1} c_{i} B_{i}(x)=c_{-1} B_{-1}(x)+c_{0} B_{0}(x)+c_{1} B_{1}(x)+ \\
\cdots+c_{N} B_{N}(x)+c_{N+1} B_{N+1}(x) .
\end{gathered}
$$

Then force $S(x)$ to satisfy the collocation equations plus the boundary conditions.

We have

$$
L_{1} S\left(x_{j}\right)=Z\left(x_{j}\right) 0 \leq x_{j} \leq \mathrm{N},
$$

and

$$
S(0)=\alpha_{0}, S(1)=\alpha_{1} .
$$

On solving Eq. (7), we get

$$
\begin{gathered}
-\epsilon S^{\prime \prime}\left(x_{j}\right)+W\left(x_{j}\right) S\left(x_{j}\right)=Z\left(x_{j}\right) \\
\Rightarrow-\epsilon \sum_{i=-1}^{N+1} c_{i} B_{i}^{\prime \prime}\left(x_{j}\right)+W\left(x_{j}\right) \sum_{i=-1}^{N+1} c_{i} B_{i}\left(x_{j}\right)=Z\left(x_{j}\right) \\
\Rightarrow-\epsilon\left[c_{j-1} B_{j-1}^{\prime \prime}\left(x_{j}\right)+c_{j} B_{j}^{\prime \prime}\left(x_{j}\right)+c_{j+1} B_{j+1}^{\prime \prime}\left(x_{j}\right)\right] \\
+W\left(x_{j}\right)\left[c_{j-1} B_{j-1}\left(x_{j}\right)+c_{j} B_{j}\left(x_{j}\right)\right. \\
\left.+c_{j+1} B_{j+1}\left(x_{j}\right)\right]=Z\left(x_{j}\right) \forall j=0,1, \ldots, N, \\
\Rightarrow c_{j-1}\left[-\epsilon B_{j-1}^{\prime \prime}\left(x_{j}\right)+W_{j} B_{j-1}\left(x_{j}\right)\right]+c_{j}\left[-\epsilon B_{j}^{\prime \prime}\left(x_{j}\right)+\right. \\
\left.W_{j} B_{j}\left(x_{j}\right)\right]+c_{j+1}\left[\left(-\epsilon B_{j+1}^{\prime \prime}\left(x_{j}\right)+W_{j} B_{j+1}\left(x_{j}\right)\right)\right]=Z_{j} \forall j= \\
0,1,2, \ldots, N,
\end{gathered}
$$

by using equations (3) and (5) we get

$$
\begin{aligned}
& \left(-6 \epsilon+W_{j} \tilde{\mathrm{h}}^{2}\right) c_{j-1}+\left(12 \epsilon+4 W_{j} \tilde{\mathrm{h}}^{2}\right) c_{j}+(-6 \epsilon+ \\
& \left.W_{j} \tilde{\mathrm{h}}^{2}\right) c_{j+1}=\tilde{\mathrm{h}}^{2} Z_{j}, \forall j=0,1, \ldots, N,
\end{aligned}
$$

where $W\left(x_{j}\right)=W_{j}$ and $Z\left(x_{j}\right)=Z_{j}$.

The given boundary conditions ( 8$)$ become 


$$
\begin{gathered}
S\left(x_{j}\right)=S\left(x_{0}\right)=\alpha_{0} \\
\Rightarrow c_{-1} B_{-1}\left(x_{0}\right)+c_{0} B_{0}\left(x_{0}\right)+c_{1} B_{1}\left(x_{0}\right)+\cdots \\
+c_{N+1} B_{N+1}\left(x_{0}\right)=\alpha_{0} \\
\Rightarrow c_{-1}+4 c_{0}+c_{1}=\alpha_{0}
\end{gathered}
$$

and

$$
\begin{gathered}
S\left(x_{j}\right)=S\left(x_{N}\right)=\alpha_{1} \\
\Rightarrow c_{-1} B_{-1}\left(x_{N}\right)+c_{0} B_{0}\left(x_{N}\right)+c_{1} B_{1}\left(x_{N}\right)+\cdots \\
+c_{N-1} B_{N-1}\left(x_{N}\right)+c_{N} B_{N}\left(x_{N}\right) \\
+c_{N+1} B_{N+1}\left(x_{N}\right)=\alpha_{1} \\
\Rightarrow c_{N-1}+4 c_{N}+c_{N+1=} \alpha_{1} .
\end{gathered}
$$

Eqs. (10), (11) and (12) lead to a $(N+3) \times(N+3)$ tridiagonal system with $(N+3)$ unknowns $C_{N}=$ $\left(c_{-1}, c_{0}, \ldots, c_{N+1}\right)^{t}$ (where $t$ stands for transpose).

Now eliminating $c_{-1}$ from the first equation of (10) and (11), we find

$$
36 \epsilon c_{0}=Z_{0} \tilde{\mathrm{h}}^{2}-\alpha_{0}\left(-6 \epsilon+W_{0} \tilde{\mathrm{h}}^{2}\right) .
$$

Similarly, eliminating $c_{N+1}$ from the last equation of (10) and from (12), we find

$$
36 \epsilon c_{N}=Z_{N} \tilde{\mathrm{h}}^{2}-\alpha_{1}\left(-6 \epsilon+W_{N} \tilde{\mathrm{h}}^{2}\right) .
$$

from (10) we get

$$
\begin{gathered}
j=1:\left(-6 \epsilon+W_{1} \tilde{\mathrm{h}}^{2}\right) c_{0}+\left(12 \epsilon+4 W_{1} \tilde{\mathrm{h}}^{2}\right) c_{1}+\left(-6 \epsilon+W_{1} \tilde{\mathrm{h}}^{2}\right) c_{2}=\mathrm{Z}_{1} \tilde{\mathrm{h}}^{2} \\
j=2:\left(-6 \epsilon+W_{2} \tilde{\mathrm{h}}^{2}\right) c_{1}+\left(12 \epsilon+4 W_{2} \tilde{\mathrm{h}}^{2}\right) c_{2}+\left(-6 \epsilon+W_{2} \tilde{\mathrm{h}}^{2}\right) c_{3}=\mathrm{Z}_{2} \tilde{\mathrm{h}}^{2} \\
\vdots \\
j=i:\left(-6 \epsilon+W_{i} \tilde{\mathrm{h}}^{2}\right) c_{i-1}+\left(12 \epsilon+4 W_{i} \tilde{\mathrm{h}}^{2}\right) c_{i}+\left(-6 \epsilon+W_{i} \tilde{\mathrm{h}}^{2}\right) c_{i+1}=\mathrm{Z}_{i} \tilde{\mathrm{h}}^{2} \\
\vdots \\
j=N-1:\left(-6 \epsilon+W_{N-1} \tilde{\mathrm{h}}^{2}\right) c_{N-2}+\left(12 \epsilon+4 W_{N-1} \tilde{\mathrm{h}}^{2}\right) c_{N-1}+\left(-6 \epsilon+W_{N-1} \tilde{\mathrm{h}}^{2}\right) c_{N}=\mathrm{Z}_{\mathrm{N}-1} \tilde{\mathrm{h}}^{2}
\end{gathered}
$$

The above equations lead to the system of $(N+1)$ linear equations $T x_{N}=d_{N}$ in the $(N+1)$ unknowns $x_{N}=\left(c_{0}, \ldots, c_{N}\right)^{t}$ of the form

$$
\left[\begin{array}{cccccc}
36 \epsilon & & & & & \\
\gamma & \beta & \gamma & & & \\
& \gamma & \beta & \gamma & & \\
& & \ddots & \ddots & \ddots & \\
& & & \gamma & \beta & \gamma \\
& & & & & 36 \epsilon
\end{array}\right]\left[\begin{array}{c}
c_{0} \\
c_{1} \\
c_{2} \\
\vdots \\
c_{N-1} \\
c_{N}
\end{array}\right]=\left[\begin{array}{c}
Z_{0} \tilde{\mathrm{h}}^{2}-\alpha_{0} \gamma \\
Z_{1} \tilde{\mathrm{h}}^{2} \\
Z_{2} \tilde{\mathrm{h}}^{2} \\
\vdots \\
Z_{N-1} \tilde{\mathrm{h}}^{2} \\
Z_{N} \tilde{\mathrm{h}}^{2}-\alpha_{1} \gamma
\end{array}\right]
$$

where

$$
\begin{aligned}
& \gamma=-6 \epsilon+W \tilde{\mathrm{h}}^{2}, \\
& \beta=12 \epsilon+4 W \tilde{\mathrm{h}}^{2} .
\end{aligned}
$$

Since $W(x)>0$, it is easily seen that the matrix $T$ is strictly diagonally dominant and hence nonsingular. Since $T$ is nonsingular, we can solve the system $T x_{N}=d_{N}$ for $c_{0}, c_{1}, \ldots, c_{N}$ and substitute into the boundary equations (11) and (12) to obtain $c_{-1}$ and $c_{N+1}$

Lemma [8] The B-splines $\left\{B_{-1}, B_{0}, \ldots, B_{N+1}\right\}$ defined in equation (2), satisfy the inequality

$$
\sum_{i=-1}^{\mathrm{N}+1}\left|\mathrm{~B}_{i}(x)\right| \leq 10,0 \leq x \leq 1
$$

Proof. We know that

$$
\left|\sum_{i=-1}^{N+1} \mathrm{~B}_{i}(x)\right| \leq \sum_{i=-1}^{N+1}\left|B_{i}(x)\right|
$$

At any $i$ th nodal point $x_{i}$ we have

$$
\sum_{i=-1}^{\mathrm{N}+1}\left|\mathrm{~B}_{i}\right|=\left|\mathrm{B}_{i-1}\right|+\left|\mathrm{B}_{i}\right|+\left|\mathrm{B}_{i+1}\right|=6<10 .
$$

Also we have

$$
\left|B_{i}(x)\right| \leq 4 \text { and }\left|B_{i-1}(x)\right| \leq 4 \text { for } x \in\left[x_{i-1}, x_{i}\right] .
$$

Similarly

$$
\left|B_{i-2}(x)\right| \leq 1 \text { and }\left|B_{i+1}(x)\right| \leq 1 \text { for } x \in\left[x_{i-1}, x_{i}\right] .
$$

Now for any point $x \in\left[x_{i-1}, x_{i}\right]$ we have

$$
\sum_{i=-1}^{N+1}\left|B_{i}(x)\right|=\left|B_{i-2}\right|+\left|B_{i-1}\right|+\left|B_{i}\right|+\left|B_{i+1}\right| \leq 10 .
$$

Hence this proves the lemma.

\section{Numerical Result}

The exact solution of problem (1) is known to be 


$$
u(x, t)=e^{-4 \pi^{2} t} \sin 2 \pi x, \mathrm{~g}(x)=\sin 2 \pi x .
$$

Denote the value of $u$ at the representative mesh point $P\left(x_{j}, t_{n}\right)$ by

$$
u_{P}=u\left(x_{j}, t_{n}\right)=u_{j}^{n}
$$

The forward difference approximation for $\frac{\partial u}{\partial t}$ is

$$
\frac{\partial u}{\partial t} \approx \frac{u_{j}^{n+1}-u_{j}^{n}}{\Delta t}
$$

Substitute $P=u_{j}$ in (16) we get

$$
\begin{gathered}
\frac{P^{n+1}(x)-P^{n}(x)}{\Delta t}=\frac{\partial^{2}}{\partial x^{2}} P^{n+1}(x) \quad-k \sum_{i=-1}^{N+1} c_{i} B_{i}^{\prime \prime}\left(x_{j}\right)+\sum_{i=-1}^{N+1} c_{i} B_{i}\left(x_{j}\right)=\mathrm{g}\left(x_{j}\right) \\
\Rightarrow-\Delta t P_{x x}^{n+1}+P^{n+1}=P^{n} \\
\Rightarrow-k\left[c_{j-1} B_{j-1}^{\prime \prime}\left(x_{j}\right)+c_{j} B_{j}^{\prime \prime}\left(x_{j}\right)+c_{j+1} B_{j+1}^{\prime \prime}\left(x_{j}\right)\right]+c_{j-1} B_{j-1}\left(x_{j}\right)+c_{j} B_{j}\left(x_{j}\right)+c_{j+1} B_{j+1}\left(x_{j}\right)=\mathrm{g}\left(x_{j}\right) \forall j=0,1, \ldots, N, \\
\Rightarrow c_{j-1}\left[-k B_{j-1}^{\prime \prime}\left(x_{j}\right)+B_{j-1}\left(x_{j}\right)\right]+c_{j}\left[-k B_{j}^{\prime \prime}\left(x_{j}\right)+B_{j}\left(x_{j}\right)\right]+c_{j+1}\left[-k B_{j+1}^{\prime \prime}\left(x_{j}\right)+B_{j+1}\left(x_{j}\right)\right]=\mathrm{g}\left(x_{j}\right) \forall j=0,1, \ldots, N, \quad(18)
\end{gathered}
$$

by using equations (3) and (5) we get

$$
\begin{gathered}
\left(-6 k+\tilde{\mathrm{h}}^{2}\right) c_{j-1}+\left(12 k+4 \tilde{\mathrm{h}}^{2}\right) c_{j}+\left(-6 k+\tilde{\mathrm{h}}^{2}\right) c_{j+1}=\mathrm{g}_{j} \tilde{\mathrm{h}}^{2} \forall \mathrm{j}=0,1, \ldots, \mathrm{N}, \\
j=1:\left(-6 k+\tilde{\mathrm{h}}^{2}\right) c_{0}+\left(12 k+4 \tilde{\mathrm{h}}^{2}\right) c_{1}+\left(-6 k+\tilde{\mathrm{h}}^{2}\right) c_{2}=\mathrm{g}_{1} \tilde{\mathrm{h}}^{2} \\
j=2:\left(-6 k+\tilde{\mathrm{h}}^{2}\right) c_{1}+\left(12 k+4 \tilde{\mathrm{h}}^{2}\right) c_{2}+\left(-6 k+\tilde{\mathrm{h}}^{2}\right) c_{3}=\mathrm{g}_{2} \tilde{\mathrm{h}}^{2} \\
\vdots \quad \text { and (12) to obtain } c_{-1} \text { and } c_{N+1} .
\end{gathered}
$$$$
j=i:\left(-6 k+\tilde{\mathrm{h}}^{2}\right) c_{i-1}+\left(12 k+4 \tilde{\mathrm{h}}^{2}\right) c_{i}+\left(-6 k+\tilde{\mathrm{h}}^{2}\right) c_{i+1}
$$$$
=\mathrm{g}_{i} \tilde{\mathrm{h}}^{2}
$$$$
\begin{aligned}
& j=N-1:\left(-6 k+\tilde{\mathrm{h}}^{2}\right) c_{N-2}+\left(12 k+4 \tilde{\mathrm{h}}^{2}\right) c_{N-1} \\
&+\left(-6 k+\tilde{\mathrm{h}}^{2}\right) c_{N}=\mathrm{g}_{\mathrm{N}-1} \tilde{\mathrm{h}}^{2}
\end{aligned}
$$

The above equations lead to the system of $(N+1)$ linear equations $T x_{N}=d_{N}$ in the $(N+1)$ unknowns $x_{N}=$ $\left(c_{0}, c_{1}, \ldots, c_{N}\right)^{t}$ of the form

$$
\left[\begin{array}{cccccc}
36 k & & & & & \\
\gamma & \beta & \gamma & & & \\
& \gamma & \beta & \gamma & & \\
& & \ddots & \ddots & \ddots & \\
& & & \gamma & \beta & \gamma \\
& & & & & 36 k
\end{array}\right]\left[\begin{array}{c}
c_{0} \\
c_{1} \\
c_{2} \\
\vdots \\
c_{N-1} \\
c_{N}
\end{array}\right]=\left[\begin{array}{c}
\mathrm{g}_{0} \tilde{\mathrm{h}}^{2} \\
\mathrm{~g}_{1} \tilde{\mathrm{h}}^{2} \\
\mathrm{~g}_{2} \tilde{\mathrm{h}}^{2} \\
\vdots \\
\mathrm{g}_{\mathrm{N}-1} \tilde{\mathrm{h}}^{2} \\
\mathrm{~g}_{N} \tilde{\mathrm{h}}^{2}
\end{array}\right],
$$

where

$$
\begin{aligned}
& \gamma=-6 k+\tilde{\mathrm{h}}^{2}, \\
& \beta=12 k+4 \tilde{\mathrm{h}}^{2} .
\end{aligned}
$$

We can see that the system is strictly diagonally dominant and hence nonsingular. So we can solve the system for $c_{0}, c_{1}, \ldots, c_{N}$ and substitute into the boundary conditions (11)
The table 2 below illustrates the numerical, exact solution and error for the heat equation $P_{r}$

Table 2. Numerical, Exact Solution And Error For The Heat Equation $P_{r}$.

\begin{tabular}{llll}
\hline $\mathbf{x}$ & $\mathbf{p} \mathbf{i}$ Numerical & U_exact & error \\
\hline 0 & 0 & 0 & 0 \\
0.0500 & 0.0108 & 0.0060 & 0.0185 \\
0.1000 & 0.0206 & 0.0113 & 0.0353 \\
0.1500 & 0.0284 & 0.0156 & 0.0486 \\
0.2000 & 0.0333 & 0.0184 & 0.0571 \\
0.2500 & 0.0351 & 0.0193 & 0.0600 \\
0.3000 & 0.0333 & 0.0184 & 0.0571 \\
0.3500 & 0.0284 & 0.0156 & 0.0486 \\
0.4000 & 0.0206 & 0.0113 & 0.0353 \\
0.4500 & 0.0108 & 0.0060 & 0.0185 \\
0.5000 & 0.0000 & 0.0000 & 0.0000 \\
0.5500 & -0.0108 & -0.0060 & 0.0185 \\
0.6000 & -0.0206 & -0.0113 & 0.0353 \\
0.6500 & -0.0284 & -0.0156 & 0.0486 \\
0.7000 & -0.0333 & -0.0184 & 0.0571 \\
0.7500 & -0.0351 & -0.0193 & 0.0600 \\
0.8000 & -0.0333 & -0.0184 & 0.0571 \\
0.8500 & -0.0284 & -0.0156 & 0.0486 \\
0.9000 & -0.0206 & -0.0113 & 0.0353 \\
0.9500 & -0.0108 & -0.0060 & 0.0185 \\
1.0000 & 0 & -0.0000 & 0.0000 \\
\hline
\end{tabular}




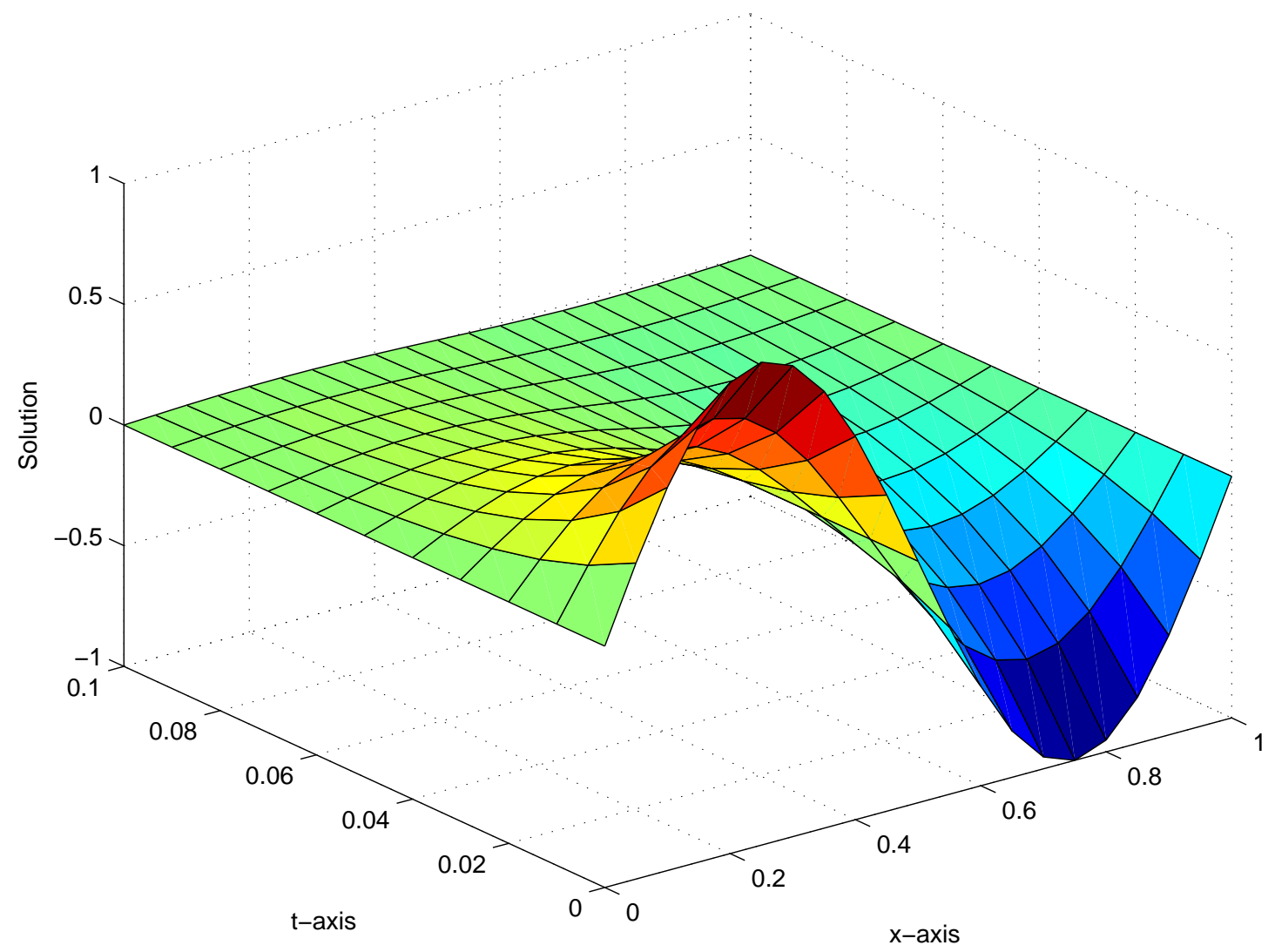

Figure 1. Exact solution for the heat problem $P_{r}$.

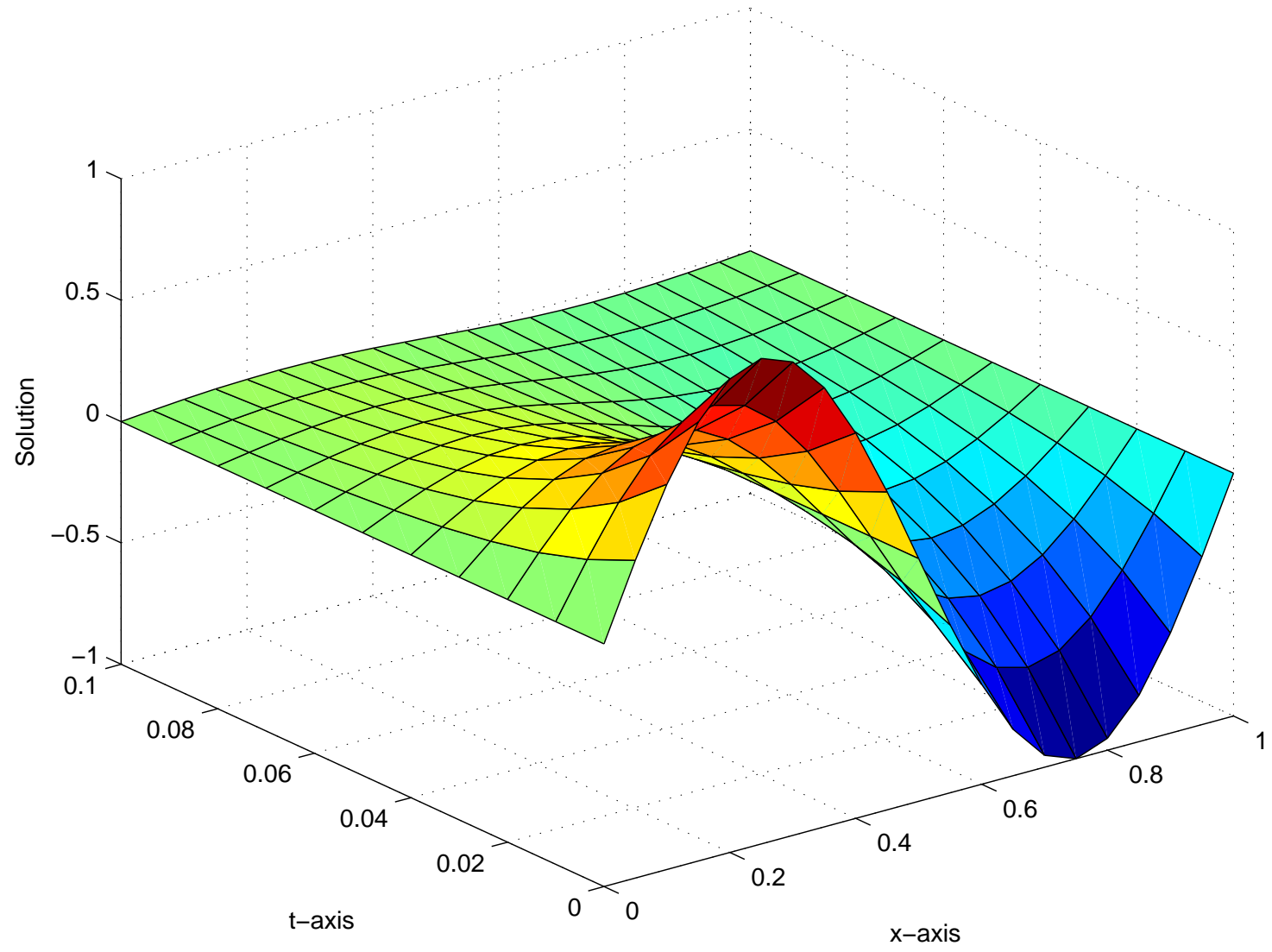

Figure 2. Numerical solution for the heat problem $P_{r}$ using $h=0.05$ and $k=0.01$. 

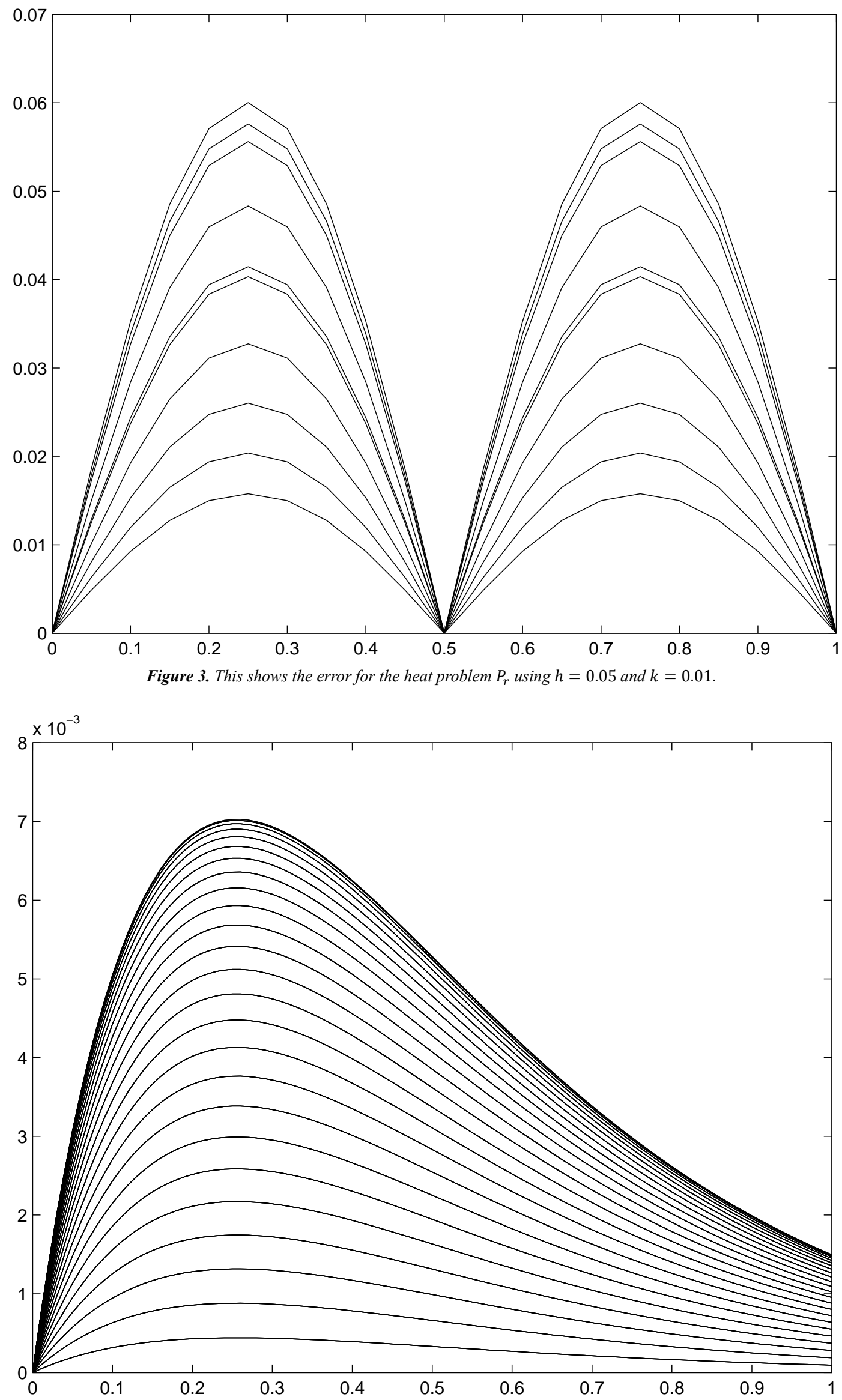

Figure 4. This shows the error for the heat problem $P_{r}$ using $h=0.01$ and $k=0.001$. 


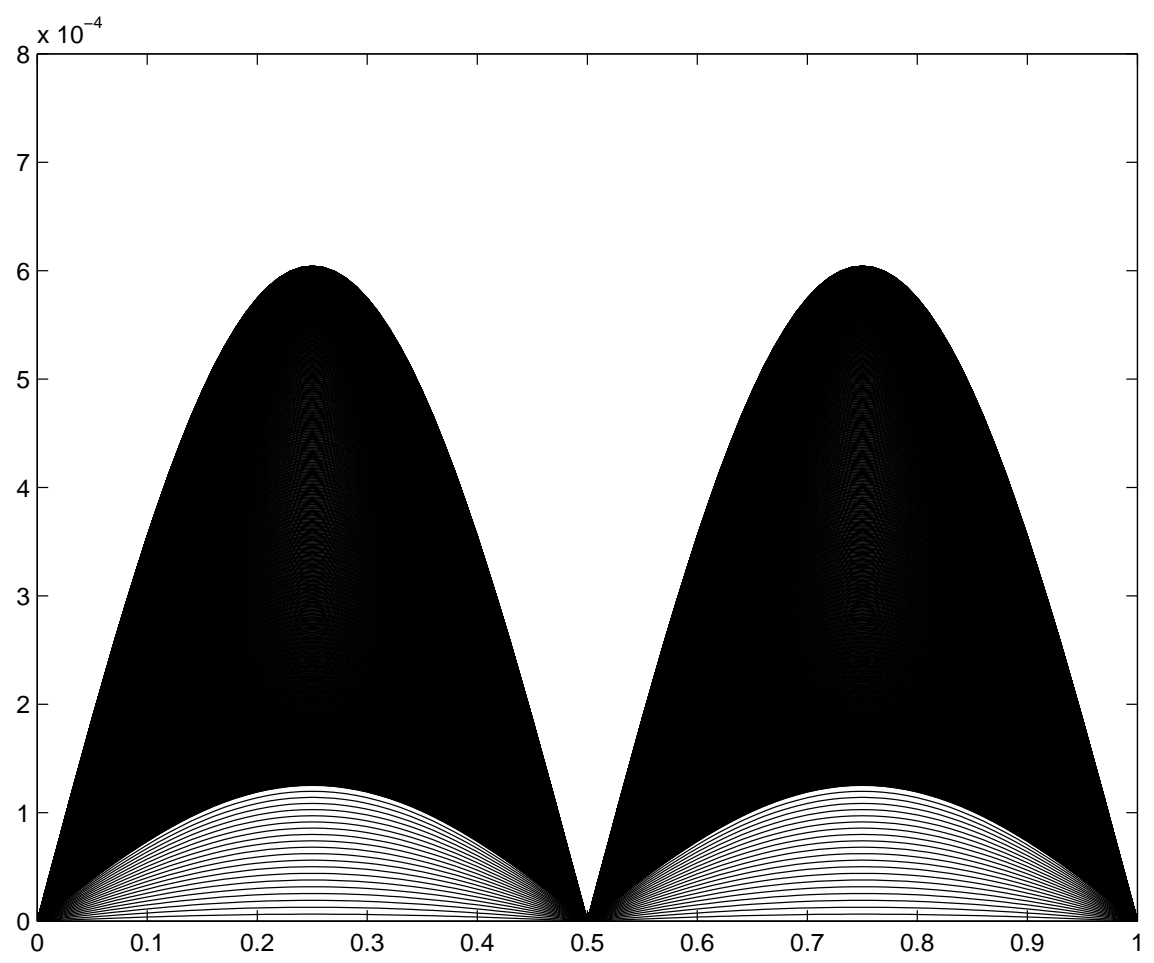

Figure 5. This shows the error for the heat problem $P_{r}$ using $h=0.01$ and $k=0.0001$.

\section{Conclusion}

We applied the cubic B-spline collocation method to solve one-dimensional heat equation. The results and graphs have also been shown using MATLAB for the comparison between the numerical and exact solutions.

Numerical experiments are conducted to demonstrate the viability and the efficiency of the proposed method computationally.

\section{Appendix}

Program for the heat problem $P_{r}$ :

Main_bspline:

clc

clear

$\mathrm{h}=0.05 ; \mathrm{x} 0=0 ; \mathrm{x} 1=1 ; \mathrm{b}=1 ; \mathrm{t} 0=0 ; \mathrm{t} 1=0.1 ; \mathrm{k}=0.01$;

$\% \mathrm{~h}=0.05 ; \mathrm{x} 0=0 ; \mathrm{x} 1=1 ; \mathrm{b}=1 ; \mathrm{t} 0=0 ; \mathrm{t} 1=0.1 ; \mathrm{k}=$ 0.001

$\% \mathrm{~h}=0.01 ; \mathrm{x} 0=0 ; \mathrm{x} 1=1 ; \mathrm{b}=1 ; \mathrm{t} 0=0 ; \mathrm{t} 1=0.1 ; \mathrm{k}=$ 0.001 ;

$\% \mathrm{~h}=0.01 ; \mathrm{x} 0=0 ; \mathrm{x} 1=1 ; \mathrm{b}=1 ; \mathrm{t} 0=0 ; \mathrm{t} 1=0.1 ; \mathrm{k}=$ 0.0001

$\% \mathrm{j}=0 ; 1 ; \ldots ; \mathrm{n}$;

$\mathrm{x}=\mathrm{x} 0: \mathrm{h}: \mathrm{x} 1$

$\operatorname{sizex}=\mathrm{x}$;

$\mathrm{t}=\mathrm{t} 0: \mathrm{k}: \mathrm{t} 1$

$\mathrm{M}=$ length(x);

$\mathrm{N}=$ length $(\mathrm{t})$

$\mathrm{p} 0=\sin \left(2 * \mathrm{pi}^{*} \mathrm{x}\right)$;

$\mathrm{u}=\operatorname{zeros}(\mathrm{N}, \mathrm{M})$;

$\mathrm{u}(1,:)=\mathrm{p} 0$;
$\% \mathrm{M}=\mathrm{M}-2$;

for $\mathrm{i}=2$ : $\mathrm{N}$

[p_i] = fun_bspline $(\mathrm{p} 0, \mathrm{~h}, \mathrm{k}, \mathrm{M})$;

$\mathrm{u}(\mathrm{i},:)=\mathrm{p}_{\text {i }}$;

p0 = p_i;

end

\%\%\%\%\%\%\%\%\%\%\%\%\%\%\%\%\%\%\%\%\%\%\%\%\%\%\%\% $\% \% \% \% \%$

$\mathrm{V}=\operatorname{zeros}(\mathrm{N}, \mathrm{M})$

$\mathrm{V}(1,:)=\sin \left(2 * \mathrm{pi}^{*} \mathrm{x}\right)$;

for $\mathrm{i}=1: \mathrm{N}$

for $\mathrm{j}=1: \mathrm{M}$

$\mathrm{V}(\mathrm{i}, \mathrm{j})=\exp \left(-4 * \mathrm{t}(\mathrm{i}) * \mathrm{pi}^{\wedge} 2\right) * \sin \left(2 * \mathrm{pi}^{*} \mathrm{x}(\mathrm{j})\right)$;

U_exact $(j)=\exp \left(-4 * t(i) * \mathrm{pi}^{\wedge} 2\right) * \sin \left(2 * \mathrm{pi}^{*} \mathrm{x}(\mathrm{j})\right)$;

end

end

error $=\max (\mathrm{abs}(\mathrm{u}-\mathrm{V}))$;

yerr $=\operatorname{abs}(\mathrm{u}-\mathrm{V})$;

$\mathrm{y}=\left[\mathrm{x}^{\prime} \mathrm{p}\right.$ _i' U_exact' error']

$\%$

Figure(1)

$\operatorname{surf}(\mathrm{x}, \mathrm{t}, \mathrm{V})$

$\%$----------------

$\%$ figure(1)

$\% \operatorname{plot}(\mathrm{x}, \mathrm{yerr})$

figure(2)

$\operatorname{surf}(\mathrm{x}, \mathrm{t}, \mathrm{u})$

$\%$

\%\% \% \% \% \% \% \% \% \% \% \% \% \% \% \% \% \% \% \% \% \% \% \% \% \% \% \% $\% \% \% \% \% \% \%$

$\%$ 


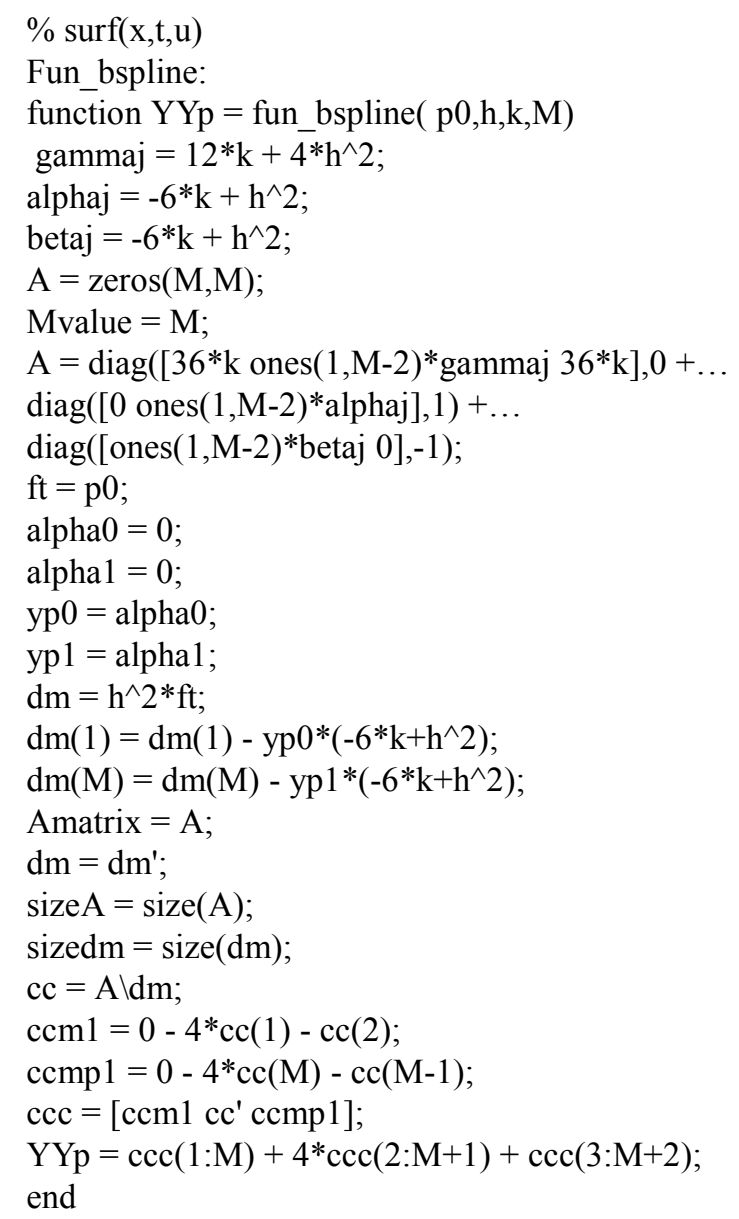

\section{References}

[1] H. S. Carslaw, J. C. Jaeger, Conduction of Heat in Solids, Oxford University Pres., 1959.

[2] I. Dag, B. Saka, D. Irk, Application Cubic B-splines for Numerical Solution of the RLW Equation, Appl. Maths. and Comp., 159 (2004) 373-389.

[3] D. V. Widder, The Heat Equation, Academic Press, 1976.

[4] J. R. Cannon, The One-Dimensional Heat Equation, Cambridge University Pres., 1984.

[5] en.wikipedia.org/wiki/Heat_equation.

[6] JBJ Fourier, Theorie analytique dela Chaleur, Didot Paris: 499-508 (1822).

[7] J. M. Ahlberg, E. N. Nilson, J. L. Walsh, The Theory of splines and Their Applications, Academic Press, New York, 1967.

[8] M. K. Kadalbajoo and V. K. Aggarwal, Fitted mesh B-spline collocation method for solving self-adjoint singularly perturbed boundary value problems, Applied Mathematics and Computation 161 (2005), 973-987.

[9] G. Micula, Handbook of Splines, Kluwer Academic Publishers, Dordrecht, The Netherlands, 1999.

[10] L. L. Schumaker, Spline Functions: Basic Theory, Krieger Publishing Company, Florida, 1981. 